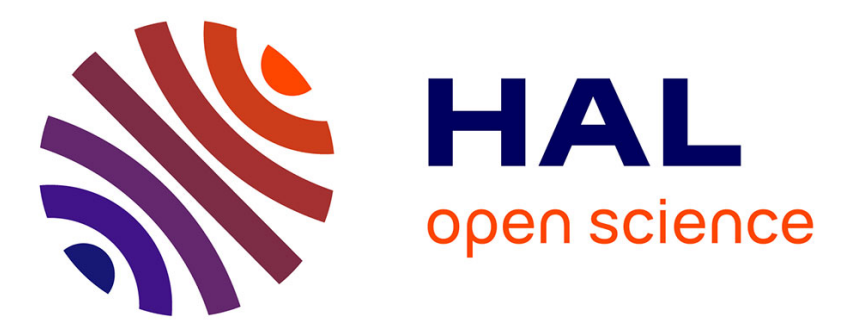

\title{
Numerical tool for SMA material simulation: application to composite structure design
}

Yves Chemisky, Arnaud Duval, Boris Piotrowski, Tarak Ben Zineb, Vanessa Tahiri, Etienne Patoor

\section{- To cite this version:}

Yves Chemisky, Arnaud Duval, Boris Piotrowski, Tarak Ben Zineb, Vanessa Tahiri, et al.. Numerical tool for SMA material simulation: application to composite structure design. Smart Materials and Structures, 2009, 18 (10), pp.104012. 10.1088/0964-1726/18/10/104012 . hal-02272048

\section{HAL Id: hal-02272048 \\ https://hal.science/hal-02272048}

Submitted on 27 Aug 2019

HAL is a multi-disciplinary open access archive for the deposit and dissemination of scientific research documents, whether they are published or not. The documents may come from teaching and research institutions in France or abroad, or from public or private research centers.
L'archive ouverte pluridisciplinaire HAL, est destinée au dépôt et à la diffusion de documents scientifiques de niveau recherche, publiés ou non, émanant des établissements d'enseignement et de recherche français ou étrangers, des laboratoires publics ou privés. 


\title{
Numerical tool for SMA material simulation: application to composite structure design
}

\author{
Yves Chemisky $^{1}$, Arnaud Duval ${ }^{2}$, Boris Piotrowski ${ }^{2}$, \\ Tarak Ben Zineb ${ }^{2}$, Vanessa Tahiri ${ }^{1}$ and Etienne Patoor ${ }^{3}$ \\ ${ }^{1}$ LPMM, Metz University, CNRS, Ile du Saulcy, F-57045 Metz Cedex 01, France \\ ${ }^{2}$ LEMTA, Nancy University, CNRS, 2 rue Jean Lamour, F-54500 Vandoeuvre-les-Nancy, \\ France \\ ${ }^{3}$ LPMM Arts et Metiers ParisTech, CNRS, 4 rue Augustin Fresnel, F-57070 Metz Cedex 01, \\ France
}

\begin{abstract}
Composite materials based on shape memory alloys (SMA) have received growing attention over these last few years. In this paper, two particular morphologies of composites are studied. The first one is an SMA/elastomer composite in which a snake-like wire NiTi SMA is embedded into an elastomer ribbon. The second one is a commercial $\mathrm{Ni}_{47} \mathrm{Ti}_{44} \mathrm{Nb}_{9}$ which presents elastic-plastic inclusions in an NiTi SMA matrix. In both cases, the design of such composites required the development of an SMA design tool, based on a macroscopic 3D constitutive law for NiTi alloys. Two different strategies are then applied to compute these composite behaviors. For the SMA/elastomer composite, the macroscopic behavior law is implemented in commercial FEM software, and for the $\mathrm{Ni}_{47} \mathrm{Ti}_{44} \mathrm{Nb}_{9}$ a scale transition approach based on the Mori-Tanaka scheme is developed. In both cases, simulations are compared to experimental data.
\end{abstract}

\section{Introduction}

More and more complex structures based on shape memory alloys (SMA) are investigated nowadays, in particular when this active material is coupled with a structural material to get a smart composite material. This is a very wide class of materials with respect to the nature of the matrix and the reinforcement but also with respect to their morphologies. A first example is the case of an SMA wire embedded in a soft matrix. But a material composed of inclusions embedded inside an SMA matrix can also be considered as a composite material. Such composites are, in fact, widely used in applications because SMA alloys are very sensitive to precipitation, and when the precipitation of a second phase occurs in an SMA material, one has then to consider a composite material to model the overall response. Of course, very different simulation tools have to be developed when we are faced with an SMA fiber embedded in a polymer matrix or with an SMA matrix with a dispersion of elastic or elastic-plastic inclusions. But, for each case, a constitutive law adapted to account in a suitable way for the behavior of the SMA materials is required. Such models have to describe correctly thermomechanical effects induced by martensitic transformation, but, when we want to apply such a law to represent the response of a complex structure, like a composite material, a compromise has to be found between the accuracy of the description of the fundamental physical mechanisms involved and a reasonable number of internal variables for memory and computation time constraints. In this paper, we choose to describe the behavior of SMA composites having different morphologies, using the same macroscopic constitutive equations for the SMA component. According to the composite morphology, very different modeling strategies will be developed. When we have to deal with an SMA fiber embedded within a polymeric 
matrix, a finite element analysis approach is adopted, and the SMA behavior law is then adapted to an FEA package. To account for the influence of elastic-plastic inclusions inside an SMA matrix we will develop a homogenization technique based on a scale transition scheme considering this macroscopic description of the SMA behavior. As the volume fraction of precipitates is low in the case considered, a Mori-Tanaka approach is very suitable. In the past few years, many macroscopic models have been developed to describe the SMA behavior [1-4]. Most of them consider two kinds of martensite: a so-called thermal martensite, with a self-accommodated structure, and a stress-oriented martensite. Macroscopic transformation strain is only due to the second one, assuming the existence of a constant transformation strain. Brinson's model [1] is focused on the orientation mechanism (transformation from thermal to stress-oriented martensite) and non-proportional loadings. Lagoudas's model [2] uses an improved pseudo-phase transition model, which differentiates transformation temperatures for each kind of martensite. This model can simulate non-proportional loadings. Lexcellent's model [3] is more focused on tension-compression asymmetry and transformation surface shape. Peultier's model [4] is based on a different choice of internal variables to describe the SMA behavior. The martensite microstructure is represented using two variables: a scalar one, the volume fraction of martensite, and a tensorial one which is the mean transformation strain. This last variable is equal to zero when the martensite was formed in a self-accommodated way and reaches a saturation value when the martensite is strongly stress-oriented by an applied stress. In the present work, we choose to deal with an improved version based on Peultier's model. This model deals with internal loops and tension-compression asymmetry specificities and considers the occurrence of a mechanical detwinning mechanism within the twinned martensite [5]. Two different composite applications are investigated using this model, which was implemented in the FEA package ABAQUS to design composite actuators made from NiTi snake-like shape wires embedded in an elastomer ribbon. To take into account the enlargement of the hysteresis size induced by the addition of elastic-plastic precipitates in NiTiNb alloys, a scale transition scheme using the Mori-Tanaka technique is adopted. The matrix behavior is then described using the macroscopic model developed and the inclusion is assumed to present an elastic-plastic behavior. This homogenization approach enables us to predict the effect of niobium precipitates in $\mathrm{NiTiNb}$ shape memory alloys for fastening applications.

\section{SMA behavior modeling}

\subsection{Kinematical description-internal variables}

The model proposed here is based, on the one hand, on a thermodynamical description of the martensitic phase transformation, reorientation and detwinning, and on the other hand on simplified micromechanical considerations. The aim is to lead to a macroscopic model based on a mesoscopic description (at the grain or single-crystal level), as proposed by Peultier et al [4]. Three internal variables are introduced. One of them is a scalar that defines the volume fraction of martensite. Both others are tensorial and define, in an averaged way, the orientation of this martensite and the accommodation of twins, respectively. A Gibbs' free energy potential is defined, including a chemical part and an interaction part between martensite variants and between grains. Driving forces related to internal variables are derived from this energy. An important point of modeling SMA is the ability to capture internal loops in thermomechanical paths. The behavior depends on the history of previous paths. Several evolution variables and memory points are introduced to design correctly internal cycles by means of time-evolving critical activation forces. The non-symmetric behavior between tension and compression is introduced through a maximum mean transformation strain depending on loading direction. This anisotropy formulation results from a micromechanical analysis [6]. The transformation strain direction is determined by a normality flow-rule assumption. All these considerations lead to nonlinear constitutive equations, solved by a NewtonRaphson algorithm. Thermomechanical tangent operators are then derived. This model is able to describe superelastic behavior, recovery stress and strain (useful to design actuators), martensite reorientation and shape memory effect. The numerical tool obtained is implemented in the ABAQUS finite element code via a UMAT subroutine. This formalism is developed in the small perturbations' framework because reversible macroscopic strain never exceeds $8 \%$. A macroscopic kinematical law can be written as an additive decomposition of the different strain mechanisms, with the assumption that austenite and martensite phases have the same elastic constants:

$$
E_{i j}=E_{i j}^{\mathrm{e}}+E_{i j}^{\mathrm{T}}+E_{i j}^{\mathrm{tw}}
$$

where

(i) $E^{\mathrm{e}}$ is the macroscopic elastic strain part.

(ii) $E^{\mathrm{T}}$ is the macroscopic transformation strain part.

(iii) $E^{\mathrm{tw}}$ is the macroscopic accommodation of the twin strain part.

$E^{\mathrm{tw}}$ is an additional inelastic strain contribution introduced in equation (1) with the transformation one. In this way, we take into account the experimental observations made in [7] about the twins accommodation process in NiTi alloys. The transformation strain part is the mean of the local transformation strain on the RVE:

$$
E_{i j}^{\mathrm{T}}=\frac{1}{V} \int_{V} \varepsilon_{i j}^{\mathrm{T}}(r) \mathrm{d} V .
$$

Assuming that the transformation strain is non-zero only in the martensite part, and considering $f$ as the volume fraction of martensite, which is the ratio of the martensite volume $V_{\mathrm{M}}$ inside the RVE volume $V$, one can write

$$
E_{i j}^{\mathrm{T}}=\frac{V_{\mathrm{M}}}{V} \frac{1}{V_{\mathrm{M}}} \int_{V_{\mathrm{M}}} \varepsilon_{i j}^{\mathrm{T}}(r) \mathrm{d} V=f \frac{1}{V_{\mathrm{M}}} \int_{V_{\mathrm{M}}} \varepsilon_{i j}^{\mathrm{T}}(r) \mathrm{d} V .
$$

The mean transformation strain in the martensite part is then defined as follows:

$$
\bar{\varepsilon}_{i j}^{\mathrm{T}}=\frac{1}{V_{\mathrm{M}}} \int_{V_{\mathrm{M}}} \varepsilon_{i j}^{\mathrm{T}}(r) \mathrm{d} V .
$$


The macroscopic transformation strain can be written as a function of $f$ and $\bar{\varepsilon}_{i j}^{\mathrm{T}}$ :

$$
E_{i j}^{\mathrm{T}}=f \bar{\varepsilon}_{i j}^{\mathrm{T}}
$$

The elastic accommodation of twins can be written using the same formalism:

$$
E_{i j}^{\mathrm{tw}}=\frac{1}{V} \int_{V} \varepsilon_{i j}^{\mathrm{tw}}(r) \mathrm{d} V .
$$

This accommodation of twins is non-zero only in martensite variants which appeared with twins inside. Considering $\gamma_{\mathrm{m}}$ as the volume fraction of martensite with twins in the RVE, and $\bar{\varepsilon}_{i j}^{\mathrm{tw}}$ as the mean accommodation strain in the martensite with twins:

$$
E_{i j}^{\mathrm{tw}}=\frac{V_{\gamma_{\mathrm{m}}}}{V} \frac{1}{V_{\gamma_{\mathrm{m}}}} \int_{V_{\gamma_{\mathrm{m}}}} \varepsilon_{i j}^{\mathrm{tw}}(r) \mathrm{d} V=\gamma_{\mathrm{m}} \bar{\varepsilon}_{i j}^{\mathrm{tw}} .
$$

The volume fraction of martensite and both the mean strain characteristic of orientation and twin accommodation mechanisms are considered in this kinematical description. The three variables $f, \bar{\varepsilon}_{i j}^{\mathrm{T}}$ and $\bar{\varepsilon}_{i j}^{\text {twin }}$ are representative of the material microstructure evolution, in the mean field formalism adopted in this paper. In a straightforward way, they are chosen as internal variables in the thermodynamical description of the transformation of the RVE.

\subsection{Thermodynamical description}

The existence of a thermodynamic potential in the two-phase RVE is postulated, and evolution laws of internal variables are derived from this potential. The first and second laws of thermodynamics are combined, leading to the ClausiusDuhem inequality:

$$
\begin{gathered}
-\frac{\partial G}{\partial \Sigma}: \dot{\Sigma}-\frac{\partial G}{\partial T} \cdot \dot{T}-\frac{\partial G}{\partial V_{k}} \cdot \dot{V}_{k}-S \cdot \dot{T} \\
-\dot{\Sigma}: E-\vec{q} \cdot \frac{\operatorname{grad} T}{T} \mathrm{~d} t \geqslant 0 .
\end{gathered}
$$

The Gibbs free energy $G$ considers the macroscopic stress $\Sigma$ and temperature $T$ as observable variables. $S$ represent the VER entropy, $E$ is the macroscopic strain and $q$ is the thermal flux. The internal variables $V_{k}$ are:

(i) $f$, the volume fraction of martensite

(ii) $\bar{\varepsilon}_{i j}^{\mathrm{T}}$, the mean martensite transformation strain

(iii) $\bar{\varepsilon}_{i j}^{\mathrm{tw}}$, the mean twin accommodation in twinned martensite variants.

Physical limitations on these internal variables must be taken into account in the Gibbs free energy evolution. From its definition, the martensite volume fraction ranges between 0 and 1. A saturated value of the mean transformation strain is introduced with a tension-compression asymmetry parameter, in order to represent the non-symmetric transformation surface according to experimental results [8, 9]. Patoor et al [6] were using a transformation yield surface formulation based on the second $\left(J_{2}\right)$ and third $\left(J_{3}\right)$ invariants of the deviatoric stress tensor. This expression was used to calculate the overall response of SMA beams [10]. Peultier et al [4] have developed a criterion based on the saturated value of the transformation strain, which depends on the second and third invariants of the mean transformation strain $\bar{\varepsilon}_{i j}^{\mathrm{T}}$. The yield stress asymmetry is also considered as the difference between the tensile and the compressive transformation strains. Peultier's criterion is here generalized to the $n$-rank with respect to convexity conditions:

$$
\bar{\varepsilon}_{\mathrm{sat}}^{\mathrm{T}}=K\left(1+\beta \frac{J_{3}}{J_{2}^{\frac{3}{2}}}\right)^{\frac{1}{n}}
$$

with

$$
J_{2}\left(\bar{\varepsilon}_{i j}^{\mathrm{T}}\right)=\frac{1}{2} \bar{\varepsilon}_{i j}^{\mathrm{T}} \bar{\varepsilon}_{i j}^{\mathrm{T}} \quad J_{3}\left(\bar{\varepsilon}_{i j}^{\mathrm{T}}\right)=\frac{1}{3} \bar{\varepsilon}_{i j}^{\mathrm{T}} \bar{\varepsilon}_{j k}^{\mathrm{T}} \bar{\varepsilon}_{k i}^{\mathrm{T}} .
$$

Parameters $K$ and $\beta$ are identified from tensile and compressive tests, considering respectively $\bar{\varepsilon}_{\text {trac }}^{\mathrm{T}}$ and $\bar{\varepsilon}_{\text {comp }}^{\mathrm{T}}$ as saturated values in tension and compression loading cases:

$$
\begin{gathered}
K=\bar{\varepsilon}_{\operatorname{trac}}^{\mathrm{T}}\left(1+\frac{\beta}{\alpha}\right)^{-\frac{1}{n}} \\
\beta=\left(\frac{1-\gamma}{\gamma+1}\right) \alpha
\end{gathered}
$$

where $\alpha$ is a constant value coming from $J_{2}$ and $J_{3}$ invariant definitions ( $\alpha \simeq 2.6$ )

$\gamma$ is the ratio of transformation strain between tension and compression at the $n$-rank:

$$
\gamma=\left(\frac{\bar{\varepsilon}_{\mathrm{comp}}^{\mathrm{T}}}{\bar{\varepsilon}_{\mathrm{trac}}^{\mathrm{T}}}\right)^{n}
$$

where $K$ and $\beta$ are characteristic parameters of the asymmetry. If $\beta=0\left(\bar{\varepsilon}_{\text {comp }}^{\mathrm{T}}=\bar{\varepsilon}_{\text {trac }}^{\mathrm{T}}\right)$, the expression of $\bar{\varepsilon}_{\text {sat }}^{\mathrm{T}}$ leads to the Von Mises equivalent strain. For common NiTi materials, the ratio between maximal compression and tension transformation strains is approximately equal to 0.8 , and $n=5$ ensures the convexity of the transformation surface.

According to experimental observations [7], accommodation of the twins process disappears at high stress levels when plastic strain occurs. The presented model is limited to elastic and transformation strain. Within the range of strains considered, one can assume that the accommodation of twin strain value is not limited. These two physical constraints are introduced by adding three Lagrange multipliers $\lambda_{i}$ in the thermodynamic potential, in order to ensure that internal variables $f$ and $\bar{\varepsilon}_{i j}^{\mathrm{T}}$ remain in their respective domains:

$$
\begin{aligned}
& \text { If } f \geqslant 0, \lambda_{0}=0 \text { and if } f<0, \lambda_{0}>0 . \\
& \text { If } f \leqslant 1, \lambda_{1}=0 \text { and if } f>1, \lambda_{1}>0 . \\
& \text { If }\left|\bar{\varepsilon}_{i j}^{\mathrm{T}}\right| \leqslant \varepsilon_{\mathrm{SAT}}^{\mathrm{T}}, \lambda_{2}=0 \text { and if }\left|\bar{\varepsilon}^{\mathrm{T}}\right|>\varepsilon_{\mathrm{SAT}}^{\mathrm{T}}, \lambda_{2}>0 .
\end{aligned}
$$

Thermoelastic balance can be defined, as well as the thermodynamical forces associated with the internal variables. The decomposition of elastic energy and linearization of entropy variation for martensitic transformation lead to the 
following equations:

- The transformation force $F_{\mathrm{f}}$ :

$$
F_{\mathrm{f}}=\Sigma_{i j} \bar{\varepsilon}_{i j}^{\mathrm{T}}-B\left(T-T_{0}\right)-H_{\mathrm{f}} f-\frac{1}{2} H_{\varepsilon}\left(\bar{\varepsilon}_{i j}^{\mathrm{T}}: \bar{\varepsilon}_{i j}^{\mathrm{T}}+\lambda_{0}-\lambda_{1} .\right.
$$

The introduction of $\lambda_{0}$ and $\lambda_{1}$ ensures that the martensite volume fraction remains inside the $[0,1]$ interval. $H_{\mathrm{f}}$ and $H_{\bar{\varepsilon}^{\mathrm{T}}}$ are two material parameters describing interactions coming from phase transformation evolution and geometrical incompatibilities. $B$ is the ClausiusClapeyron slope in the $(\sigma, T)$ diagram. $T_{0}$ is the equilibrium temperature.

- The orientation force $F_{\varepsilon^{\mathrm{T}}}$, considering $\Sigma^{\mathrm{D}}$ as the deviatoric part of the macroscopic stress $\Sigma$ :

$$
F_{\varepsilon_{i j}^{\mathrm{T}}}=\Sigma_{i j}^{\mathrm{D}}-H_{\varepsilon} \bar{\varepsilon}_{i j}^{\mathrm{T}}-\lambda_{2} .
$$

The introduction of $\lambda_{2}$ ensures that the mean transformation strain will never exceed its saturated value.

- The twin accommodation force $F_{\varepsilon}^{\mathrm{tw}}$ :

$$
F_{\varepsilon_{i j}^{\mathrm{twin}}}=\Sigma_{i j}^{\mathrm{D}}-H_{\mathrm{tw}} \bar{\varepsilon}_{i j}^{\mathrm{tw}} .
$$

The elastic balance is defined assuming that equilibrium is always realized before any dissipative mechanism:

$$
\begin{gathered}
\frac{\partial G}{\partial \Sigma_{i j}}-E_{i j}=0 \\
S_{i j k l} \Sigma_{k l}+f \bar{\varepsilon}_{i j}^{\mathrm{T}}+\gamma_{\mathrm{m}} \bar{\varepsilon}_{i j}^{\mathrm{twin}}-E_{i j}=0 .
\end{gathered}
$$

With $S_{i j k l}$ is the fourth-order compliance tensor. The definition of a dissipation potential leads to the evolution laws of the internal variables:

For the transformation force:

- If $\left|F_{\mathrm{f}}\right|<F_{\mathrm{f}}^{\text {crit }}$ or $\left|F_{\mathrm{f}}\right|=F_{\mathrm{f}}^{\text {crit }}$ and $\left|\frac{\mathrm{d}\left|F_{\mathrm{f}}\right|}{\mathrm{d} t}\right| \leqslant 0$, the force is not activated. In this case, the associated variable is constant so $\dot{f}=0$.

- If $\left|F_{\mathrm{f}}\right|=F_{\mathrm{f}}^{\text {crit }}$ and $\left|\frac{\mathrm{d}\left|F_{\mathrm{f}}\right|}{\mathrm{d} t}\right|>0$, the force is activated. In this case $\dot{f} \neq 0$.

For the orientation force: an associated rule is considered, so the 'yield' direction is normal to the orientation surface:

$$
\dot{\bar{\varepsilon}}_{i j}^{\mathrm{T}}=\dot{\lambda} \frac{\partial\left|F_{\varepsilon_{i j}^{\mathrm{T}}}\right|}{\partial F_{\varepsilon_{i j}^{\mathrm{T}}}}
$$

$F_{\varepsilon_{i j}^{\mathrm{T}}}$ is a thermodynamical force, which has a stress dimension. The asymmetry between tension and compression is included in the expression of the saturated transformation strain value. The Von Mises equivalent stress is then adopted for the expression of the orientation force norm:

$$
\left|F_{\varepsilon_{i j}^{\mathrm{T}}}\right|=\overline{\frac{3}{2} F_{\varepsilon_{i j}^{\mathrm{T}}} F_{\varepsilon_{i j}^{\mathrm{T}}}} .
$$

The orientation yield surface is defined as follows:

(i) If $\left|F_{\varepsilon_{i j}^{\mathrm{T}}}\right|<F_{\varepsilon^{\mathrm{T}}}^{\text {crit }}$ or $\left|F_{\varepsilon_{i j}^{\mathrm{T}}}\right|=F_{\varepsilon^{\mathrm{T}}}^{\text {crit }}$ and $\left|\frac{\mathrm{d}\left|F_{\varepsilon_{i j}^{\mathrm{T}}}\right|}{\mathrm{d} t}\right| \leqslant 0$ there is no orientation. In this case, if the domain does not evolve, $\dot{\bar{\varepsilon}}_{i j}^{\mathrm{T}}=0$. Otherwise, if $\dot{f}>0$, the mean transformation strain evolves in order to respect $\dot{E}_{i j}^{\mathrm{T}}=0$. Indeed, as the orientation criteria is not activated, the new martensitic domain appears with a self-accommodated structure, so the macroscopic transformation strain is not modified.

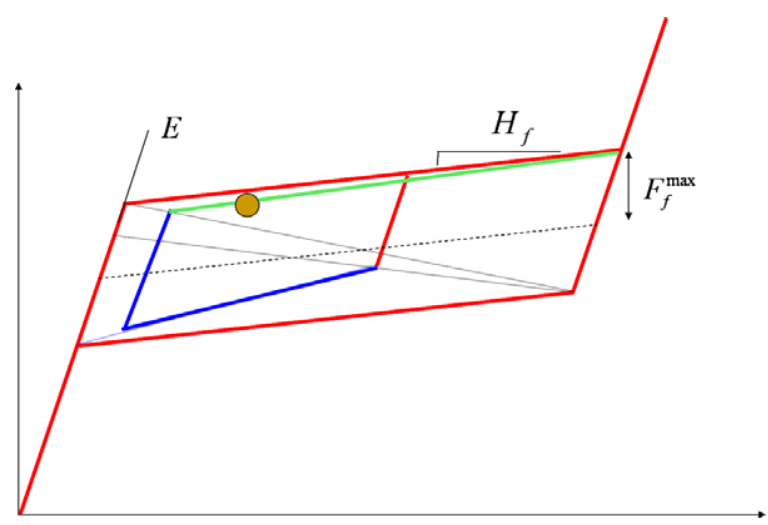

Figure 1. Inner loops in a superelastic path.

(ii) If $\left|F_{\varepsilon_{i j}^{\mathrm{T}}}\right|=F_{\varepsilon^{\mathrm{T}}}^{\mathrm{crit}}$ and $\left|\frac{\mathrm{d}\left|F_{\varepsilon_{i j}^{\mathrm{T}}}\right|}{\mathrm{d} t}\right|>0$ the orientation force is active. In this case $\dot{\bar{\varepsilon}}_{i j}^{\mathrm{T}} \neq 0$.

For the twin accommodation force: $\left|F_{\varepsilon_{i j}}\right|=0$ (the dissipation of this mechanism is neglected), so $\left|\frac{\mathrm{d}\left|F_{\varepsilon_{i j}^{\mathrm{twi}}}\right|}{\mathrm{d} t}\right|=0$ and the associated variable $\dot{\bar{\varepsilon}}_{i j}^{\text {twin }}$ will evolve in order to respect this equilibrium.

$\gamma_{\mathrm{m}}$ is a function of the mean transformation strain and the volume fraction of martensite $\gamma_{\mathrm{m}}\left(f, \bar{\varepsilon}_{\mathrm{eq}}^{\mathrm{T}}, \varepsilon_{\mathrm{SAT}}^{\mathrm{T}}\right)$, with $\bar{\varepsilon}_{\text {eq }}^{\mathrm{T}}=$ $\left|\bar{\varepsilon}_{i j}^{\mathrm{T}}\right|$ :

- If $\dot{f}>0$, then $\mathrm{d} \gamma_{\mathrm{m}}=\mathrm{d} f\left(\frac{\varepsilon_{\mathrm{SAA}}^{\mathrm{T}}-\bar{\varepsilon}_{\mathrm{eq}}^{\mathrm{T}}}{\varepsilon_{\mathrm{SAT}}^{\mathrm{T}}}\right)$.

- If $\dot{f}<0$, then $\mathrm{d} \gamma_{\mathrm{m}}=\frac{f+\mathrm{d} f}{f} \gamma_{\mathrm{m}}$.

The equivalent transformation strain is expressed using the Von Mises definition:

$$
\left|\bar{\varepsilon}_{i j}^{\mathrm{T}}\right|=\sqrt{\frac{2}{3} \bar{\varepsilon}_{i j}^{\mathrm{T}} \bar{\varepsilon}_{i j}^{\mathrm{T}}}
$$

A macroscopic SMA model must be able to capture the internal loop responses of the thermomechanical loading paths. A history dependence of the simulated behavior is introduced, with thermodynamical considerations. This formulation in the superelastic case is shown in figures 1 and 2 . The transformation criterion $\left|F_{\mathrm{f}}\right| \leqslant F_{\mathrm{f}}^{\text {crit }}$ is modified by the addition of memory force and an evolution variable that takes values in the $[0,1]$ interval. This evolution variable takes zero value at the beginning of a new loading path and is equal to 1 when the loading path reaches the saturation state.

$F_{\mathrm{f}}^{\text {mem }}$ is the value of the thermodynamic force observed at the last loading switch point. $F_{\mathrm{f}}^{\min }$ and $F_{\mathrm{f}}^{\max }$ are material parameters. A loading switch point is detected as soon as the following condition is satisfied:

$$
\left(F_{\mathrm{f}}-\left(1-\gamma_{\mathrm{f}}\right) F_{\mathrm{f}}^{\mathrm{mem}}\right)\left(f-f^{\mathrm{mem}}\right)<0
$$

where $f^{\mathrm{mem}}$ is the martensite volume fraction observed at the last loading switch point. When a loading switch point is detected, $F_{\mathrm{f}}^{\text {mem }}$ is set to $F_{\mathrm{f}}-F_{\mathrm{f}}^{\text {min }}$ and $\gamma_{\mathrm{f}}$ is reset to zero. During the loading, $\gamma_{\mathrm{f}}$ is expressed as

$$
\gamma_{\mathrm{f}}=\frac{\left|f-f^{\mathrm{mem}}\right|}{f^{\text {obj }}-f^{\mathrm{mem}}}
$$




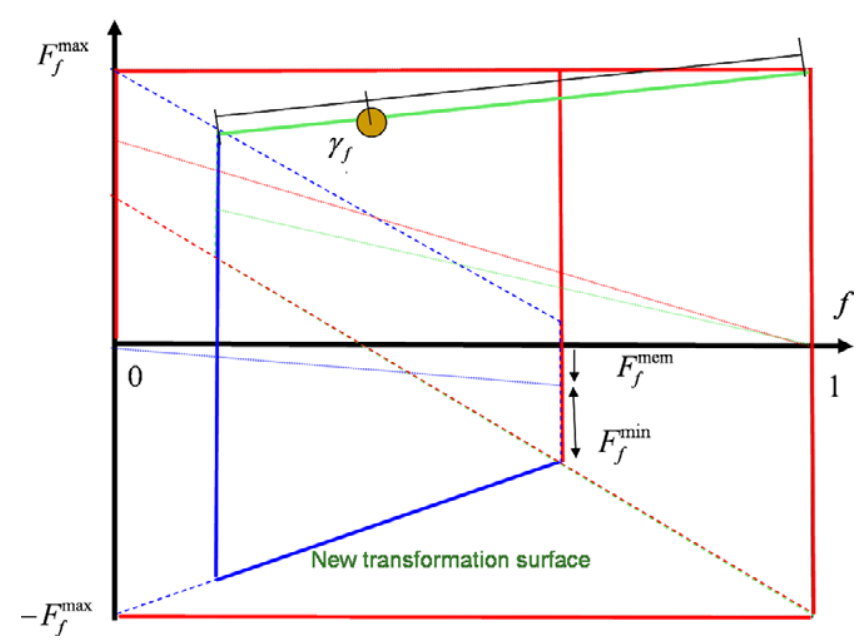

Figure 2. Evolution of $\gamma_{\mathrm{f}}$ for a superelastic path.

where $f^{\text {obj }}$ is the martensite volume fraction that can be obtained if the loading path reaches the saturation point ( 1 for direct transformation and 0 for reverse transformation).

\section{Numerical solution}

The model is implemented in such a way that it will be easily used with most commercial FEA packages, or with a scale transition scheme like the Mori-Tanaka method. The NewtonRaphson algorithm is adopted to solve the system obtained by the equilibrium condition of thermodynamical forces. The variables of the system can be divided in two parts:

- increments of driving variables: $\Delta E_{i j}$ and $\Delta T$

- increments of solution variables: $\Delta \Sigma, \Delta f, \Delta \bar{\varepsilon}_{i j}^{\mathrm{T}}, \Delta \lambda_{\bar{\varepsilon}^{\mathrm{T}}}$ and $\Delta \lambda_{\bar{\varepsilon}^{\mathrm{tw}}}$.

In each increment, a driving variable perturbation unbalances the system. The Newton-Raphson algorithm iterates to find the solution variable increment that balances the following system:

$$
\begin{gathered}
\left|F_{\mathrm{f}}-\left(1-\gamma_{\mathrm{f}}\right) F_{\mathrm{f}}^{\mathrm{mem}}\right|=F_{\mathrm{f}}^{\mathrm{min}}+\left(1-\gamma_{\mathrm{f}}\right) F_{\mathrm{f}}^{\mathrm{max}} \\
\left|F_{\bar{\varepsilon}_{i j}^{\mathrm{T}}}\right|=F_{\bar{\varepsilon}^{\mathrm{T}}}^{\mathrm{crit}} \\
\Delta \bar{\varepsilon}_{i j}^{\mathrm{T}}=\Delta \lambda_{\bar{\varepsilon}^{\mathrm{T}}} \frac{3}{2} \frac{F_{\bar{\varepsilon}_{i j}^{\mathrm{T}}}}{\left|F_{\bar{\varepsilon}_{i j}^{\mathrm{T}}}\right|} \quad\left|F_{\bar{\varepsilon}_{i j}^{\mathrm{tw}}}\right|=0 \\
\Delta \bar{\varepsilon}_{i j}^{\mathrm{tw}}=\Delta \lambda_{\bar{\varepsilon}^{\mathrm{tw}}} \frac{3}{2} \frac{\Sigma_{i j}-\frac{\Sigma_{k k}}{3} \delta_{i j}}{\Sigma_{i j}-\frac{\Sigma_{k k}}{3} \delta_{i j}} \\
E_{i j}-S_{i j k l} \Sigma_{k l}-f \bar{\varepsilon}_{i j}^{\mathrm{T}}-\gamma_{\mathrm{m}} \bar{\varepsilon}_{i j}^{\mathrm{tw}}=0 .
\end{gathered}
$$

A prediction is made using Hooke's law and, with a return mapping approach, the effective behavior is determined. The equation system is simplified if one or more mechanisms are deactivated. The SIMULA+ Mathematical Object Library is used for tensor definition and some mathematical operations [11].

\section{Implementation in the FE code ABAQUS}

The ABAQUS FE code offers the possibility to define a specific material behavior using the subroutine UMAT (User MATerial), where the user has to define the increment of stress and internal variables due to an increment of total strain and temperature. This condition justifies the choice made here to use the total strain as a driving variable instead of the stress. An interface is developed in order to convert the implemented model variables definitions to ABAQUS requirements, according to some differences in variables' storage strategies (for example, SIMULA + uses a two-dimensional table to store a symmetric tensor and ABAQUS uses a one-dimensional table with Voigt formalism). The user coding must also return a mechanical tangent operator $\frac{\partial \Delta \Sigma_{i j}}{\partial \Delta E_{k l}}$, which has to be coherent with the solving algorithm. To ensure this condition, it is extracted from the Jacobian matrix provided by the NewtonRaphson algorithm. A summary of the solution strategy is illustrated in figure 3 .

\section{Application to composite materials and structure design}

\subsection{Numerical simulation of an SMA/elastomer composite response}

When an SMA actuator is embedded in an elastomer, structural interactions between the components appear. Such effects were revealed by photoelasticimetry in a study of a snake-like NiTi wire/elastomer ribbon composite where the superelasticity effect was involved [12]. In the case presented here, both initial martensitic and austenitic states are considered, then superelasticity and reorientation behaviors are simulated. In order to develop such SMA/elastomer applications, it is important to take into account these interactions with an FEM approach and to use convenient constitutive laws for both components. For the SMA part, a macroscopic constitutive law is used. A temperature-dependent elastic law is adopted to model the behavior of the elastomer part. A $0.75 \mathrm{~mm}$ diameter nearly equiatomic NiTi wire (48.9 at.\% Ni), cold-worked at $44.7 \%$, was formed into a snake-like shape. To keep this shape and obtain thermomechanical properties such as shape memory effect and superelasticity, the snake-like wire was annealed at $450{ }^{\circ} \mathrm{C}$ for $20 \mathrm{~min}$. NiTi snakes were embedded in $12 \mathrm{~mm} \times 2 \mathrm{~mm}$ elastomer ribbons. The elastomer was chosen according to its stiffness and temperature range of use. The aim is to obtain stiffnesses of the same order of magnitude for the elastomer and for the snake wire. The elastomer used is an ALLRANE PX 14325 polyurethane produced by the Allrim Company. An ageing treatment at $40^{\circ} \mathrm{C}$ for $48 \mathrm{~h}$ was applied to the specimens, leading to stable elastomer properties. The global shape of the composite is shown in figure 4 . In order to perform the modeling of the SMA, one needs transformation temperatures, maximum transformation strain in the tensile loading path and some pseudo-hardening parameters of the NiTi wire. Tensile tests in austenite initial state (above $A_{\mathrm{f}}$ ) and martensitic state (below $M_{\mathrm{f}}$ ), cooling at constant stress and differential scanning calorimetry are also performed on straight 


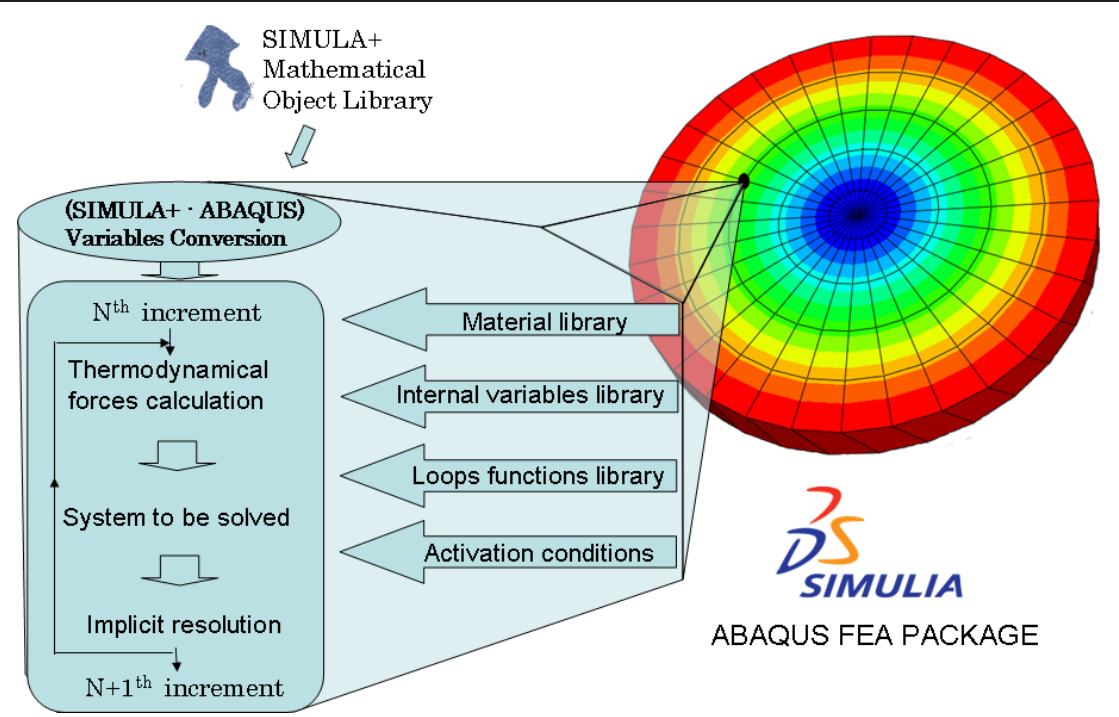

Figure 3. Sketch of the numerical strategy adopted.

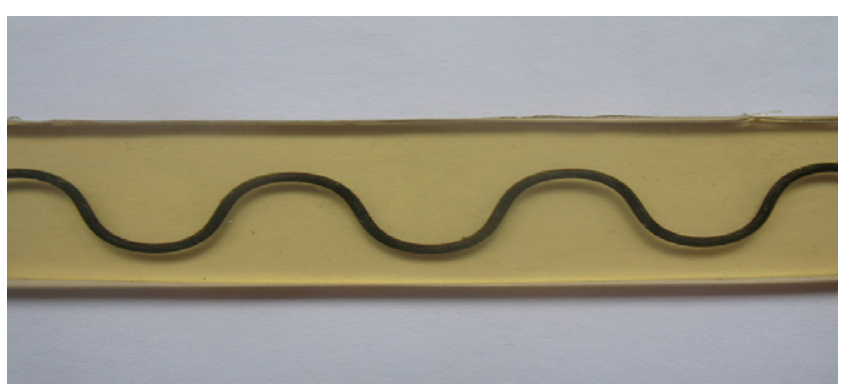

Figure 4. SMA/elastomer composite ribbon.

Table 1. Material parameters of the NiTi wire for the SMA model.

\begin{tabular}{llll}
\hline$E(\mathrm{MPa})$ & $v$ & $\varepsilon_{\text {tensile } \operatorname{Tax}}^{\mathrm{T}}$ & $\varepsilon_{\text {comp max }}^{\mathrm{T}}$ \\
55900 & 0.3 & 0.05 & 0.04 \\
\hline$B\left(\mathrm{MPa}^{\circ} \mathrm{C}^{-1}\right)$ & $M_{\mathrm{S}}\left({ }^{\circ} \mathrm{C}\right)$ & $A_{\mathrm{f}}\left({ }^{\circ} \mathrm{C}\right)$ & $r_{\mathrm{f}}$ \\
5 & -10 & 48 & 0.3 \\
\hline$F_{\varepsilon}(\mathrm{MPa})$ & $H_{\mathrm{f}}(\mathrm{MPa})$ & $H_{\varepsilon} \mathrm{T}(\mathrm{MPa})$ & $H_{\mathrm{twin}}(\mathrm{MPa})$ \\
150 & 2.2 & 650 & 8000 \\
\hline
\end{tabular}

wires (annealed in the same conditions) to find these material parameters. Parameters for the SMA NiTi wire are presented in table 1.

The ABAQUS numerical model is presented in figure 5. The circular section of the wire is modeled with an equivalent rectangular section having the same surface and the same quadratic momentum values. This allows us to use hexahedral elements for the whole structure. Complex heterogeneous stress fields impose the use of a complete 3D model to obtain accurate responses. A previous study pointed out that such fields are present in the vicinity of the SMA/elastomer interface [12]. Therefore, the wire as well as the areas near this interface are meshed more finely.

Comparison between experimental tensile tests and simulation, at various temperatures, is shown in figure 6 . From 23 to $80^{\circ} \mathrm{C}$, the material behavior is either superelastic

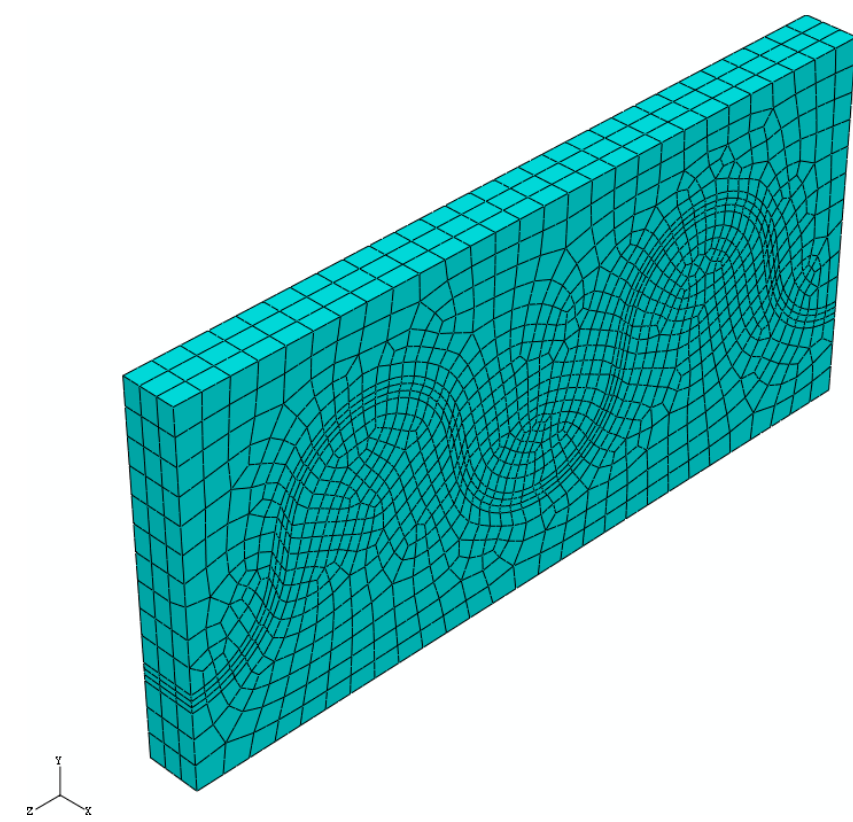

Figure 5. Finite element mesh of the composite.

or with residual transformation strain. Material parameters were identified from 80 to $-60^{\circ} \mathrm{C}$ tensile tests and from DSC experiments. At room temperature, two plateaus are observed: the first one corresponds to the R-phase transformation and the second one to the stress-induced martensite transformation. Because the R-phase transformation is not taken into account in the present model, a significant discrepancy is observed between experimental and simulated responses for lower strain levels (less than 1.5\%). However, it can be noted that superelasticity and martensite reorientation behaviors are well described by the model. The SMA/elastomer composite behavior is simulated using the ABAQUS FEM code. Figure 7 shows a comparison between numerical and experimental tensile tests at different temperatures $\left(23\right.$ and $\left.80^{\circ} \mathrm{C}\right)$. Simulated 


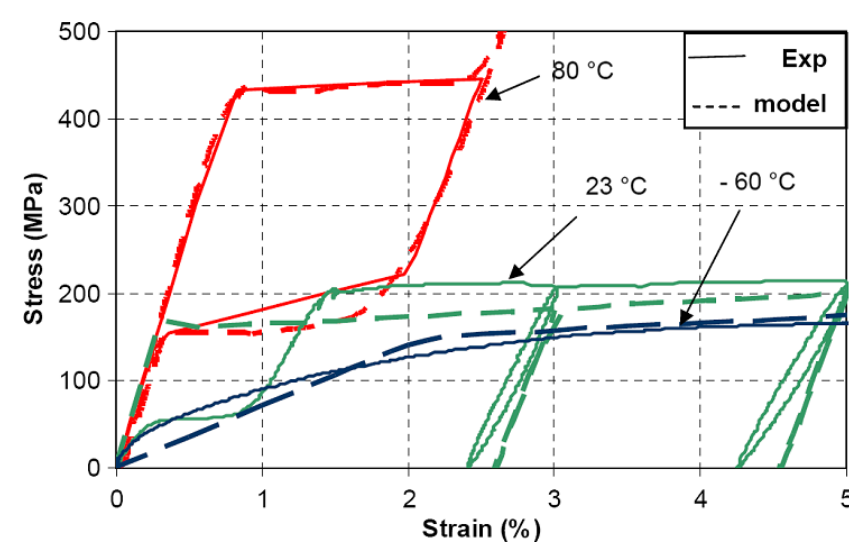

Figure 6. NiTi wire tensile behavior at various temperatures.

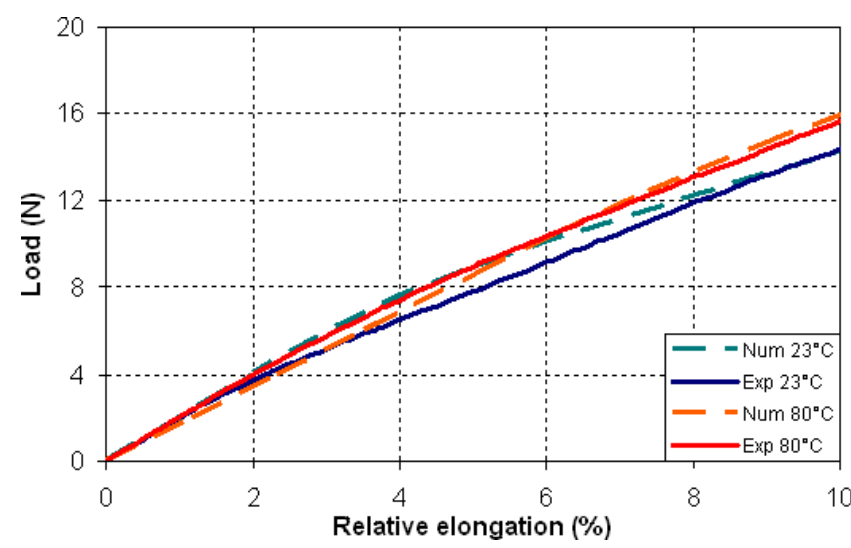

Figure 7. Comparison between experimental response and numerical simulation for the SMA/elastomer ribbon.

and experimental responses are very close for the $80^{\circ} \mathrm{C}$ isothermal test. But for the $23{ }^{\circ} \mathrm{C}$ isothermal test, one observes that the simulation overestimates the composite behavior from $4 \%$ to $8 \%$ relative elongation. In both cases, FEM results show that martensite is only present at the top of the loops, on the edges of the wire, with non-symmetric values of $f$ in the tensile or compressive fibers. This asymmetry is induced by transformation surface asymmetry and structural effects present in a curved beam. The transformation is in an advanced state for the $23^{\circ} \mathrm{C}$ simulation, but small areas are nearly fully transformed (with more than $75 \%$ martensite).

\subsection{Effect of $N b$ in NiTiNb-numerical simulation}

Commercial $\mathrm{Ni}_{47} \mathrm{Ti}_{44} \mathrm{Nb}_{9}$ shape memory alloy is generally adopted for fastening applications thanks to its wide transformation hysteresis, compared with classical NiTi. Due to its large hysteresis, at room temperature the material can be either in austenite or in martensite phase according to its previous loading history, i.e. austenite when cooled from high temperature or martensite when heated from low temperature. It has been shown that the reverse transformation temperature $A_{s}$ can increase by $80^{\circ} \mathrm{C}$ under tensile loading in the martensitic state [13]. This phenomenon is attributed to the addition of niobium: scanning electron microscopy
Table 2. Plastic parameter values for niobium inclusions.

\begin{tabular}{lllll}
\hline$E(\mathrm{MPa})$ & $v$ & $\sigma_{\mathrm{Y}}(\mathrm{MPa})$ & $H(\mathrm{MPa})$ & $n$ \\
\hline 85000 & 0.3 & 90 & 700 & 4 \\
\hline
\end{tabular}

(SEM) and x-ray diffraction (XRD) observations showed the presence of ellipsoidal-shaped niobium-rich precipitates in NiTiNb according to observations by Zhang et al [14]. This phase has been studied to quantify what kind of inclusions increases reverse transformation temperatures, and what are its properties. Nanoindentation tests revealed that niobiumrich precipitates have an elastic-plastic behavior with a low yield stress. In order to optimize the performance fastener made from this alloy, we consider $\mathrm{NiTiNb}$ as a composite material having elastic-plastic $\mathrm{Nb}$ inclusions embedded in an NiTi matrix. A homogenization scheme using the MoriTanaka model [15] is considered. The Mori-Tanaka method is one of the few explicit scale transition schemes that is suitable to save computational cost in structural analysis. Moreover, in our case, we consider a composite with a low concentration of spherical inclusions and an isotropic behavior for both inclusions and the matrix. In such a case, it was established that the Mori-Tanaka solution coincides with the upper Hashin-Shtrikman bound when the inclusion is softer than the matrix [16]. Numerical results corresponding to this approach for the composite are investigated.

In order to lead to the thermomechanical constitutive law, the NiTi matrix is described using the model presented in the first part of this paper. The constitutive equation can be written as follows:

$$
\dot{\sigma}_{i j}^{\mathrm{M}}(r)=L_{i j k l}^{\mathrm{M}}(r) \dot{\varepsilon}_{k l}^{\mathrm{M}}(r)-M_{i j}^{\mathrm{M}} \dot{T}
$$

where $L^{\mathrm{M}}$ and $M^{\mathrm{M}}$ are respectively the mechanical and thermal tangent operators and $M$ stands for the matrix.

$\mathrm{Nb}$-rich inclusions are assumed to present an elasticplastic behavior. An algorithm for plasticity developed by Simo and Hughes [17] has been adopted to model inclusions' behavior, using the Von Mises yield condition (radial return method, originally proposed by Wilkins in 1964). A swift hardening law is considered for isotropic hardening:

$$
\sigma_{\mathrm{eq}}=\sigma_{\mathrm{Y}}+H_{\mathrm{iso}} p^{\frac{1}{n}}
$$

where $\sigma_{\mathrm{Y}}$ is the plastic yield stress, $p$ is the cumulated plastic strain, and $H_{\text {iso }}$ and $n$ are two hardening parameters. These last have been identified on a tensile test curve on pure niobium samples [18], whose corresponding material parameters are presented in table 2.

The inclusion constitutive equation can be written as follows:

$$
\dot{\sigma}_{i j}^{\mathrm{I}}(r)=L_{i j k l}^{\mathrm{I}}(r) \dot{\varepsilon}_{k l}^{\mathrm{I}}(r)-C_{i j k l}^{\mathrm{I}}(r) \alpha_{k l}^{\mathrm{I}} \dot{T}
$$

where $L^{\mathrm{I}}$ and $\alpha^{\mathrm{I}}$ are respectively the mechanical and thermal tangent operators, $C^{\mathrm{I}}$ the elasticity tensor, and I stands for inclusion.

Experimental study shows that $\mathrm{Nb}$-rich inclusions are present in a relatively small fraction (10\%), the matrix 


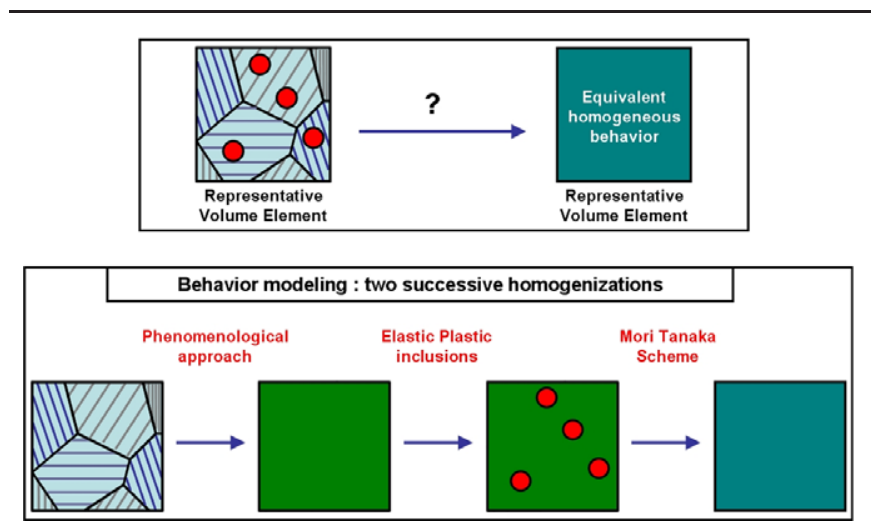

Figure 8. Homogenization strategy for NiTiNb global behavior determination.

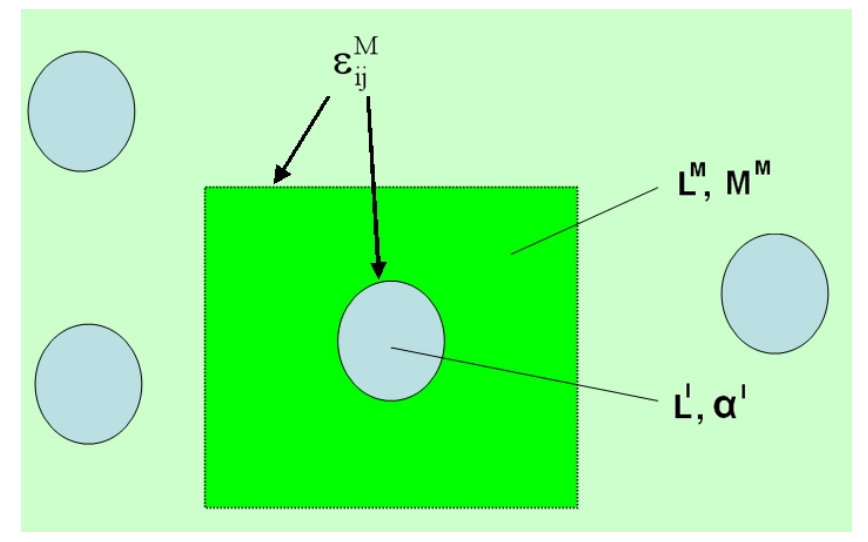

Figure 9. Elastic-plastic Mori-Tanaka solution.

is well defined and inclusions are well distributed. For this kind of microstructure, stress concentration and strain inhomogeneities associated with the inelastic behavior are not very pronounced when we consider spherical inclusions, and the Mori-Tanaka scheme gives a very good estimation of the solution for isotropic media and for a volume fraction of inclusions up to $30 \%$ [19]. This approach is then adopted to lead to the effective behavior, starting from matrix and inclusion behavior, as illustrated in figure 8 . The strain rate on the boundary of the inclusion is assumed to be equal to the strain rate in the matrix $[20,21]$, as illustrated in figure 9:

$$
\dot{\varepsilon}_{i j}^{\mathrm{I}}=\dot{\varepsilon}_{i j}^{\mathrm{M}}+T_{i j k l}\left(L_{k l m n}^{\mathrm{M}}-L_{k l m n}^{\mathrm{I}}\right) \dot{\varepsilon}_{m n}^{\mathrm{I}} .
$$

$T$ is a fourth-order interaction tensor derived from Eshelby inclusion theory. The global strain rate is assumed to be the sum between inclusion strain rate and matrix strain rate, each one being pondered by its volume fraction $\left(f^{\mathrm{I}}\right.$ is the volume fraction of inclusions):

$$
\dot{E}_{i j}=f^{\mathrm{I}} \dot{\varepsilon}_{i j}^{\mathrm{I}}+\left(1-f^{\mathrm{I}}\right) \dot{\varepsilon}_{i j}^{\mathrm{M}} .
$$

The concentration fourth-order tensor is introduced:

$$
\dot{\varepsilon}_{m n}^{\mathrm{I}}=A_{m n i j} \dot{E}_{i j} .
$$

From equations (27) and (28) we get

$$
A_{m n i j}=\left[I_{i j m n}-\left(1-f^{\mathrm{I}}\right) T_{i j k l}\left(L_{k l m n}^{\mathrm{M}}-L_{k l m n}^{\mathrm{I}}\right)\right]^{-1} .
$$

Table 3. Material parameter values for the Ni-Ti matrix.

\begin{tabular}{llllll}
\hline$E$ & $v$ & $\bar{\varepsilon}_{i j}^{\text {trac }}$ & $\bar{\varepsilon}_{i j}^{\text {comp }}$ & $M_{\mathrm{s}}$ & \\
70000 & 0.3 & 0.05 & 0.039 & -101 & \\
\hline$F_{\varepsilon}$ & $A_{\mathrm{f}}$ & $b$ & $H_{\text {var }}$ & $H_{\text {grain }}$ & $H_{\text {twin }}$ \\
160 & 63 & 5.9 & 5 & 2100 & 8800 \\
\hline
\end{tabular}

Table 4. Plastic parameter values for niobium inclusions.

\begin{tabular}{lllll}
\hline$E(\mathrm{MPa})$ & $v$ & $\sigma_{\mathrm{Y}}(\mathrm{MPa})$ & $H(\mathrm{MPa})$ & $n$ \\
\hline 85000 & 0.3 & 90 & 700 & 1 \\
\hline
\end{tabular}

The strain inside matrix is determined as

$$
\dot{\varepsilon}_{i j}^{\mathrm{M}}=\frac{1}{1-f^{\mathrm{I}}}\left(\left(I_{i j m n}-f^{\mathrm{I}} A_{i j m n}\right) \dot{E}_{m n}\right) .
$$

The strain in each phase can derived from macroscopic strain (29) and (31). So, by taking into account the constitutive equations for inclusion and matrix (26) and (24), the effective constitutive law is derived as follows:

$$
\dot{\Sigma}_{i j}=L_{i j m n}^{\mathrm{eff}} \dot{E}_{m n}-M_{i j}^{\mathrm{eff}} \dot{T} .
$$

with

$$
\begin{gathered}
L_{i j m n}^{\mathrm{eff}}=f^{\mathrm{I}}\left(L_{i j k l}^{\mathrm{I}}-L_{i j k l}^{\mathrm{M}}\right) A_{k l m n}+L_{i j m n}^{\mathrm{M}} \\
M_{i j}^{\mathrm{eff}}=f^{\mathrm{I}} C_{i j k l}^{\mathrm{I}} \alpha_{k l}^{\mathrm{I}}+\left(1-f^{\mathrm{I}}\right) M_{i j}^{\mathrm{M}} .
\end{gathered}
$$

We apply this homogenization scheme to describe two loading cases: a tension-compression one and a shape memory sequence on an SMA containing $20 \%$ of elastic-plastic inclusions. Material parameters are chosen using experimental results, and are presented in table 3 for the SMA matrix and in table 4 for niobium inclusions, considered to have a constant hardening so that $n=1$. Figure 10 represents the evolution of effective stress with effective strain for the tension-compression loading. The variation of stress with strain in each phase is also illustrated.

During the tensile part of the loading, the effective stress evolution can be decomposed into three stages. In the first one, both matrix and inclusions behave elastically. At $30 \mathrm{MPa}$, the inclusion stress reaches the niobium critical yield stress, so the plasticity is activated. Consequently, the effective behavior is affected. When effective stress exceeds $100 \mathrm{MPa}$, the matrix stress reaches the transformation critical stress and the stressinduced transformation occurs, leading to the third stage of loading.

During the compression part, three stages are also observed. The first one is related to elasticity in each phase until $125 \mathrm{MPa}$ for the effective stress. At this stress level, the stress in the elastic-plastic inclusion is about $-120 \mathrm{MPa}$ and the plasticity is activated. The second and third stages are linked to reorientation of the martensite until the saturation of reorientation.

Finally, in the following tension loading, three stages appear, respectively corresponding to elasticity, plasticity into inclusions and martensite reorientation. It is worth noting that the plastic phenomenon induces a hysteresis enlargement: indeed, at the same effective strain level of $4 \%$, the effective 


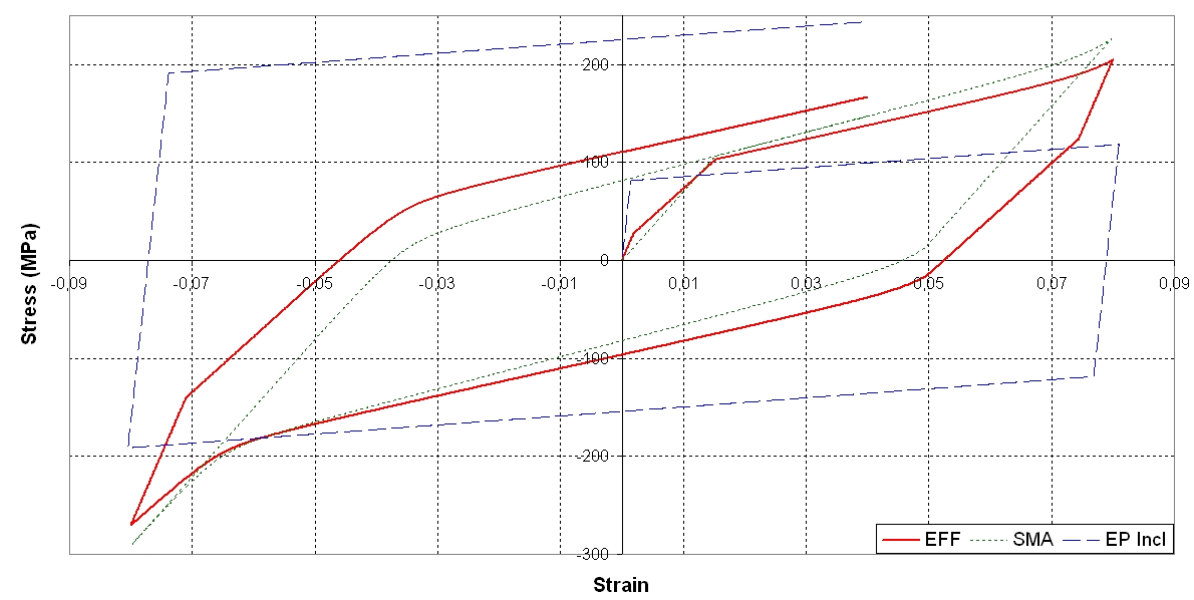

Figure 10. Tension-compression cycles for (1) effective behavior of NiTiNb, (2) SMA matrix response and (3) niobium inclusions.

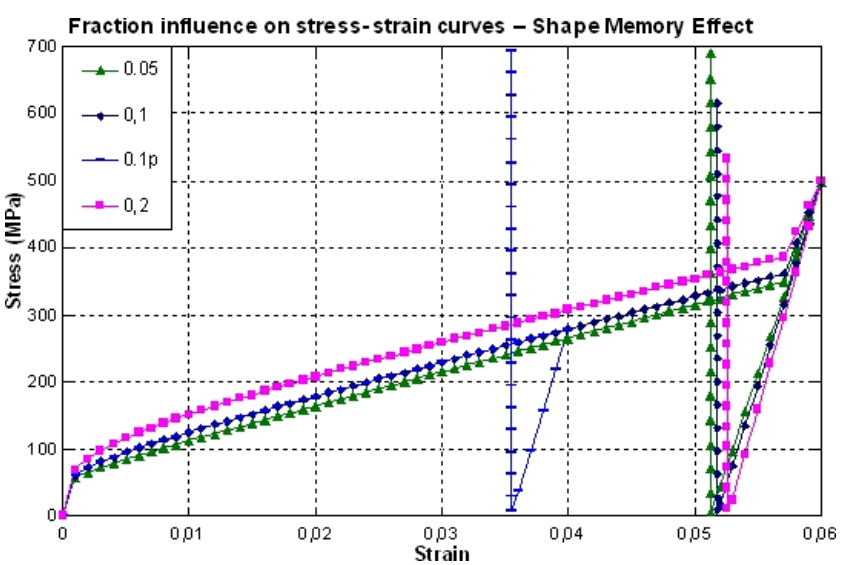

Figure 11. Strain-stress results.

stress is not the same in the first $(138 \mathrm{MPa})$ and second (167 MPa) cycles whereas the matrix stress value is the same.

In the second case, a shape memory loading is applied, constituted by tension at a temperature below $M_{\mathrm{f}}$ followed by unloading until the zero stress level, then a heating at constant strain. Simulations are made with various inclusion fractions and results are shown in figures 11 and 12 . The temperature has been decreased to $-20^{\circ} \mathrm{C}$, then an effective strain of $6 \%$ has been applied. After unloading until zero stress level, heating has been applied until $200^{\circ} \mathrm{C}$.

Figure 11 shows that the inclusions' fraction has no impact on the effective critical orientation stress. When the stress in the matrix reaches the critical orientation stress of martensite variants, an increase of effective stress results in a higher stress increase in the inclusions. These last ones will consequently easily come into the plastic domain, which explains that matrix martensitic orientation and inclusion plastic strain start at the same point.

The inclusion fraction strongly influences the effective stress developed on heating for constraint recovery. The recovery stress decreases when the inclusion amount increases.

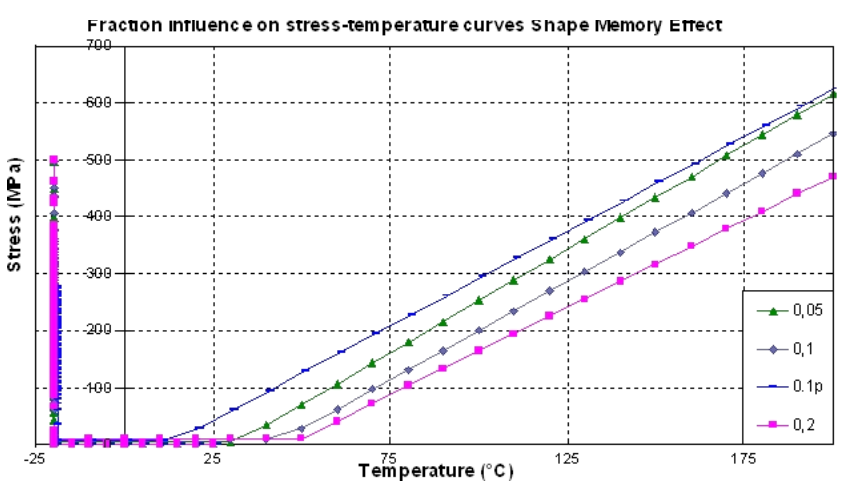

Figure 12. Stress-temperature results.

The third and most interesting point is the comparison between cases where the imposed effective strain is not the same. In the case of $10 \%$ inclusions, two loadings have been applied: the first up to a strain of $6 \%$ and the second up to a strain of $4 \%$ (respectively ' 0.1 ' and ' $0.1 p$ ' in figures 11 and 12). A predeformation imposed at the martensitic state permits us to increase the reverse starting temperature. Figure 12 shows that the higher the strain during loading, the higher the reverse transformation starting temperature. In the first case, this temperature is equal to $40{ }^{\circ} \mathrm{C}$ whereas it is about $10^{\circ} \mathrm{C}$ in the second case. To analyze the influence of the inclusion shape, a finite element analysis has to be performed in order to compare it with predictions obtained by the Mori-Tanaka scheme.

\section{Conclusion}

Two applications of SMA composite design are presented. For each of them, the same constitutive macroscopic law for the SMA part is developed. This model describes transformation, orientation and accommodation of the twins mechanism. Moreover, tension-compression asymmetry and internal loops paths are taken into account. The model is implemented in a finite element code to simulate thermomechanical paths applied to composite structures. This tool is used to describe the behavior of an SMA/elastomer composite, and 
the comparison with experiments shows that such structures can be simulated for various loadings when SMA is either in the martensite or austenite state. Heterogeneous stress fields induced by structural interactions are in accordance with previous photoelasticimetry observations [12]. A modified version of the model, including a Mori-Tanaka scale transition scheme, is developed in order to describe the influence of elastic-plastic niobium inclusions in commercial NiTiNb alloys. This modified model will be used to design industrial applications by means of a finite element code. Further work in the field of composite structures as well as materials containing inclusions is necessary. One of them is the improvement of the SMA constitutive law by including the R-phase transformation which occurs in NiTi SMAs. Experimental tests for industrial applications are also necessary to validate the simulation's capability for complex loading paths. Finally, microstructural observations and comparison with micromechanical models will lead to a better knowledge of strain mechanisms present in SMA-based material, to upgrade macroscopic predictions in the field of SMA smart structures.

\section{Acknowledgments}

The authors acknowledge the financial support of the French National Agency for Research (ANR), through the MAFESMA French and European project (Mathematical Algorithm Finite Element shape memory alloys). We also acknowledge Schlumberger Company for the financial support of NiTiNb project development.

\section{References}

[1] Bekker A and Brinson L 1998 Phase diagram based description of the hysteresis behavior of shape memory alloys Acta Mater. 46 3649-65

[2] Popov P and Lagoudas D 2007 A 3D constitutive model for shape memory alloys incorporating pseudoelasticity and detwinning of self-accommodated martensite Int. J. Plast. 23 1679-720

[3] Thiebaud F, Lexcellent C, Collet M and Foltete E 2007 Implementation of a model taking into account the asymmetry between tension and compression, the temperature effects in a finite element code for shape memory alloys structures calculations Comput. Mater. Sci. 41 208-21

[4] Peultier B, Ben Zineb T and Patoor E 2006 Macroscopic constitutive law of shape memory alloy thermomechanical behaviour. Application to structure computation by fem Mech. Mater. 38 510-24
[5] Chemisky Y, Duval A, Ben Zineb T and Patoor E 2009 Shape memory alloys modeling, including phase transformation and twinning effects, submitted

[6] Patoor E, Amrani M E, Eberhardt A and Berveiller M 1995 Determination of the origin for the dissymmetry observed between tensile and compression tests on shape memory alloys J. Physique IV 5 495-500

[7] Liu Y, Xie Z, Humbeeck J V and Delaey L V 1999 Some results on the detwinning process in NiTi shape memory alloys Scr. Mater. 41 1273-81

[8] Bouvet C, Calloch S and Lexcellent C 2004 Implementation of a model taking into account the asymmetry between tension and compression, the temperature effects in a finite element code for shape memory alloys structures calculations Eur. $J$. Mech. A 23 37-61

[9] Lexcellent C, Viver A, Bouvet C, Calloch S and Blanc P 2002 Experimental and numerical determinations of the initial surface of phase transformation under biaxial loading in some polycrystalline shape-memory alloys J. Mech. Phys. Solids $\mathbf{5 0} 2717-35$

[10] Gillet Y, Patoor E and Berveiller M 1998 Calculation of pseudoelastic elements using a non-symmetrical thermomechanical transformation criterion and associated rules J. Intell. Mater. Syst. Struct. 9 366-78

[11] Simula+ http://www.univ-valenciennes.fr/lamav/Simula+/

[12] Chemisky Y, Tahiri V, Eberhardt A and Patoor E 2007 Numerical modelling of a shape memory alloy/elastomer composite MHM, ECCOMAS Thematic Conf.

[13] He X and Rong L 2004 Dsc analysis of reverse martensitic transformation in deformed $\mathrm{Ti}-\mathrm{Ni}-\mathrm{Nb}$ shape memory alloy Scr. Mater. 51 7-11

[14] Zhang C, Wang Y, Chai W and Zhao L 1991 The study of constitutional phases in a $\mathrm{Ni}_{47} \mathrm{Ti}_{44} \mathrm{Nb}_{9}$ shape memory alloy Mater. Chem. Phys. 28 43-50

[15] Mori T and Tanaka K 1973 Average stress in matrix and average elastic energy of materials with misfitting inclusions Acta Metall. 21 571-4

[16] Hu G K and Weng G J 2000 Some reflections on the Mori-Tanaka and Ponte Casteñada-Willis methods with randomly oriented ellipsoidal inclusions Acta Mech. 140 31-40

[17] Simo J and Hughes T 2000 Computational Inelasticity (Berlin: Springer)

[18] Nemat-Nasser S and Guo W 2000 Flow stress of commercially pure niobium over a broad range of temperatures and strain rates Mater. Sci. Eng. A 284 202-10

[19] Chaboche J L, Kanouté P and Roos A 2005 On the capabilities of mean-field approaches for the description of plasticity in metal matrix composites Int. J. Plast. 21 1409-34

[20] Kröner E 1977 Bounds for effective elastic moduli of disordered materials J. Mech. Phys. Solids 25 137-55

[21] Eshelby J 1961 Elastic inclusion and inhomogeneities Prog. Solid Mech. 289 